\title{
( \\ Murray Valley encephalitis: a review of clinical features, diagnosis and treatment
}

\begin{abstract}
James Knox BSc(Med), MBBS, DTM\&H Infectious Disease Registrar $^{1}$

Raquel U Cowan

MB BS, PhD, Infectious Diseases Registrar $^{2}$

Joseph S Doyle MB BS, MSC, FRACP Infectious Diseases Physician $^{3}$

Matthew K

Ligtermoet

MB BS, BMed Sci, Neurology Registrar ${ }^{2}$

John S Archer MB BS, FRACP, PhD Neurologist ${ }^{4}$

James N C Burrow

MB BS, FRACP,

Neurologist ${ }^{5}$

Steven Y C Tong

MB BS, FRACP, PhD,

Infectious Diseases Physician $^{5,6}$

Bart J Currie FRACP, FAFPHM, DTM\&H Professor in Medicine ${ }^{5,}$

John S Mackenzie

PhD, FASM, FACTM Professor, ${ }^{7}$ and Honorary Senior Principal Fellow

David W Smith

MB BS, FRCPA, FACTM Clinical Professor ${ }^{9}$

Mike Catton BSc(Hons), MB ChB, FRCPA Director

Rodney J Moran Manager $^{10}$

Craig A Aboltins MB BS(Hons), FRACP. Infectious Diseases Physician $^{2}$

Jack S Richards MB BS, FRACP, PhD, Infectious Diseases Physician

continued next pag

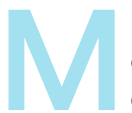
urray Valley encephalitis virus (MVEV) is a mosquito-borne virus. It is a member of the Japanese encephalitis serological complex of flaviviruses, which includes Japanese encephalitis virus (JEV), West Nile virus (WNV), Kunjin virus (KUNV), and others causing encephalitis worldwide. Major outbreaks of MVEV occurred in Australia in 1951, 1956 and 1974, with the virus first being isolated during the 1951 outbreak. ${ }^{1}$ MVEV may have caused earlier outbreaks of Australian " $X$ " disease in 1917-18, 1922 and 1925. ${ }^{2}$ In the most recent outbreak of MVEV in 1974, 58 cases of encephalitis were identified, ${ }^{3}$ indicating the significance of this disease despite the infrequency of epidemics.

MVEV is thought to be maintained in enzootic foci in the north of Western Australia and the Top End of the Northern Territory in a cycle involving waterbirds and mosquitoes, primarily the species Culex annulirostris. Most cases in humans since 1974 have occurred in these areas. Activity outside the enzootic areas is believed to follow the migration of infected waterbirds into flood-affected areas. ${ }^{4,5}$ MVEV also infects a wide range of native and nonnative animals, ${ }^{6}$ but the role of these species in maintenance and transmission of the virus is unclear. MVEV is also endemic to Papua New Guinea and Irian Jaya. ${ }^{7}$

The sporadic nature of MVEV infection in south-eastern Australia has led to the development of several models attempting to predict epidemics. These include the hypotheses of Forbes ${ }^{8}$ and Nicholls, ${ }^{9}$ which are based on patterns of rainfall and other climatic conditions. ${ }^{3}$ The predictive value of these models has been difficult to ascertain because of the infrequency of MVEV activity in south-eastern Australia, but it is likely that factors other than just flooding are required for epidemics. Challenges in prevention and control of MVEV also include difficulties in controlling mosquito numbers during periods of extensive flooding, and a lack of other prophylactic or treatment measures for MVEV.
\end{abstract}

Online first $23 / 01 / 12$

\section{Recent circumstances}

MVEV was detected along the Murray River in February 2011 through widespread seroconversion among sentinel chicken flocks after heavy rainfall and regional flooding. High numbers of mosquitoes were also observed, with $C$. annulirostris predominating. In 2011, there were increased numbers of cases of MVEV in humans across Australia, including nine cases (one death) from Western Australia, four cases (one death) from the Northern Territory, two cases (one death) from South Australia, one case from New

\begin{abstract}
Summars
Murray Valley encephalitis virus (MVEV) is a mosquitoborne virus that is found across Australia, Papua New Guinea and Irian Jaya.

MVEV is endemic to northern Australia and causes occasional outbreaks across south-eastern Australia. 2011 saw a dramatic increase in MVEV activity in endemic regions and the re-emergence of MVEV in south-eastern Australia.

This followed significant regional flooding and increased numbers of the main mosquito vector, Culex annulirostris, and was evident from the widespread seroconversion of sentinel chickens, fatalities among horses and several cases in humans, resulting in least three deaths.

The last major outbreak in Australia was in 1974, during which 58 cases were identified and the mortality rate was about 20\%.

With the potential for a further outbreak of MVEV in the 2011-2012 summer and following autumn, we highlight the importance of this disease, its clinical characteristics and radiological and laboratory features.

We present a suspected but unproven case of MVEV infection to illustrate some of the challenges in clinical management.

It remains difficult to establish an early diagnosis of MVEV infection, and there is a lack of proven therapeutic options.
\end{abstract}

South Wales, and one suspected but unproven case from Victoria (see case history below). ${ }^{10}$ MVEV was also detected in horses exhibiting neurological symptoms across south-eastern Australia. Although the immediate risk subsided with the onset of winter, it is important that doctors consider MVEV infection in patients with a suggestive clinical picture and who have been in regions of known MVEV activity. In this review, we discuss current knowledge of the clinical features of MVEV infection, challenges in its diagnosis, and the limitations in treatment.

\section{Report of a suspected case of Murray Valley encephalitis virus (MVEV) infection in Victoria}

A 69-year-old man from the Murray River town of Mildura presented to hospital in early March 2011 with a 3-day history of agitation, a 1-day history of confusion, rightsided weakness, ataxia, nausea and vomiting on a background of early Alzheimer dementia. A history of numerous mosquito bites in the preceding weeks was elicited from the family. Examination showed a mild right hemiparesis, ataxic gait and a Glasgow Coma Score (GCS) of 13/15 (verbal response, 4; eye opening, 4; motor response, 5). He was initially afebrile, and had a normal white cell count and C-reactive protein level. A chest $\mathrm{x}$-ray showed 
right upper-lobe changes, and brain computed tomography with contrast showed no acute abnormality. He was treated with aspirin, ceftriaxone and azithromycin for a presumed ischaemic stroke and pneumonia.

Over the ensuing 12 hours, the patient became febrile $\left(39^{\circ} \mathrm{C}\right)$ and increasingly confused and agitated. He then had a cardiac arrest, but was successfully cardioverted and intubated. Cerebrospinal fluid (CSF) examination on Day 5 of symptoms showed a white cell count of $6 \times 10^{6} / \mathrm{L}$ (reference interval $\left.[\mathrm{RI}],<5 \times 10^{6} / \mathrm{L}\right)$, an erythrocyte count of $7 \times 10^{6} / \mathrm{L}\left(\mathrm{RI}, 0 \times 10^{6} / \mathrm{L}\right)$, a protein concentration of $1.11 \mathrm{~g} / \mathrm{L}$ (RI, $0.15-0.45 \mathrm{~g} / \mathrm{L}$ ) and a glucose concentration of $4.6 \mathrm{mmol} /$ L (RI, 2.5-5.0 $\mathrm{mmol} / \mathrm{L}$ ); there was insufficient sample volume to measure the white cell differential. A gram stain and bacterial culture were negative. Acyclovir and benzylpenicillin were added to his antibiotic regimen to cover possible herpes simplex virus (HSV) and Listeria monocytogenes infection. The patient was then transferred to an intensive care unit. Brain stem dysfunction was shown by a GCS of 3 (while not sedated), lack of spontaneous ventilation, blood pressure instability, flaccid paralysis, occasional side-toside nystagmus, an absent gag and vestibulo-ocular reflexes. An electroencephalogram showed diffuse slowing consistent with encephalopathy. Therapy with dexamethasone was started, and the antibiotic cover broadened to include meropenem and vancomycin.

Magnetic resonance imaging (MRI) of the brain on Day 9 of symptoms showed confluent high signal bilaterally in the thalami, brainstem and the left precentral gyrus (Box 1). There was no evidence of ischaemic stroke, and findings on magnetic resonance angiography were unremarkable. Flavivirus-group and MVEV-specific reverse transcription polymerase chain reaction (PCR) findings were negative on CSF (Day 5, CSF diluted 1:2 because of the small volume available), and on plasma (Day 8). PCRs on CSF for alphavirus and herpes virus (types 1 and 2, Epstein-Barr virus and cytomegalovirus) were also negative. Flavivirus IgG was detected in serum by immunofluorescence (IF) on Day 4 at a titre of 1:640, but IF for flavivirus IgM and an MVEV-specific epitope-blocking enzymelinked immunosorbent assay (ELISA) were negative. A Day 8 serum sample tested at another reference laboratory by epitope-blocking ELISA (targeting a different antigenic region $^{11}$ ) showed $M V E V-$ specific antibodies at a titre of $>1$ : 320 , and was confirmed by a test for MVEV-specific neutralising antibodies. The results of IF for MVEV IgM remained negative. Other investigations did not show an alternative diagnosis. Unfortunately, the patient showed no clinical improvement and brain death was pronounced on Day 10. Treatment was withdrawn and an autopsy request was declined.

\section{Clinical features}

It is estimated that between 1 in 150 and 1 in 1000 MVEV infections result in symptomatic disease. ${ }^{6,12}$ People of all ages are affected and the clinical features comprise an incubation period of 1-4 weeks (average, 2 weeks), then a prodrome of 2-5 days that usually includes fever (often $>40^{\circ} \mathrm{C}$ ) and headache. ${ }^{13-18}$ Other prodromal features may include nausea, vomiting, diarrhoea, macular rash and cough. ${ }^{15,17}$ Neurological features occur early and may

1 Fluid attenuation inversion recovery magnetic resonance imaging of the brain of a 69-year-old man from flood-affected regional Victoria

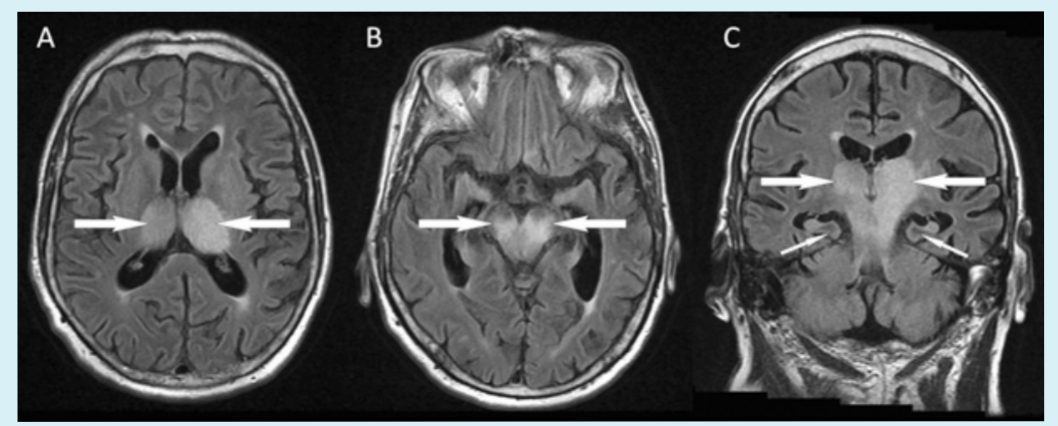

Axial images ( $A$ and $B$ ) show diffuse high signal bilaterally in the thalami, cerebral peduncles and midbrain (bold arrows). The coronal image (C) shows confluent high signal changes extending from the thalami to the midbrain and pons (bold arrows). The small hippocampi noted bilaterally were consistent with early Alzheimer disease (small arrows).

include lethargy, irritability and confusion. Seizures almost invariably occur in children, and may also occur in adults. Progression of neurological features varies, and four clinical patterns have been described, including: relentless progression to death; prominent spinal cord involvement causing flaccid paralysis; cranial nerve/brainstem involvement and tremor; and encephalitis followed by complete recovery. ${ }^{17}$ Mild cases of encephalitis not requiring hospitalisation, and non-encephalitic cases with fever and headache have also been described, suggesting a broad spectrum of disease. ${ }^{15}$ The clinical presentations of infection with different encephalitic flaviviruses may overlap, and clinical features cannot reliably differentiate between these diseases. Nonetheless, JEV may be more likely to cause Parkinsonian features, cranial nerve, cerebellar and upper motor neuron signs, ${ }^{19}$ and WNV may cause flaccid paralysis (suggesting an anterior horn cell process similar to poliomyelitis), seizures, cerebellar ataxia, brachial plexopathy and optic neuritis. ${ }^{19}$ The case fatality rate of MVEV is about $15 \%-30 \%, 16,18$ with long-term neurological sequelae occurring in $30 \%-50 \%$ of survivors and only $40 \%$ recovering completely. ${ }^{14-18}$ The pathogenic processes mediating susceptibility to the disease are unknown, and it remains unclear whether the higher incidence of disease among Aboriginal people and males reflects exposure or a specific pathogenic process. ${ }^{13-17}$ There are also no clearly defined features that predict subsequent clinical outcomes, although these appear to be worse in those with flaccid paralysis, and in the young or the elderly. ${ }^{16,17}$

\section{Radiological features}

Radiological imaging makes a critical contribution to an early diagnosis of MVEV infection, often providing supportive information before serological results are available and helping to exclude alternative diagnoses. MRI is the most sensitive and specific type of imaging, and may show positive findings within a week of symptom onset. Typical features include bilateral hyperintensity of the deep grey matter, especially the thalami, on fluid attenuation inversion recovery or $\mathrm{T} 2$-weighted images (Box 1). These changes may also involve the temporal lobes, red nucleus and cervical spinal cord, and, in some cases, they may resemble those seen in HSV encephalitis. ${ }^{20}$ Findings may be similar for other flaviviruses, especially JEV and WNV, from previous page

1 Victorian Infectious Diseases Service, Roya Melbourne Hospital, Melbourne, VIC.

2 The Northern Hospital Melbourne, VIC.

3 Victorian Infectious Diseases Reference Laboratory, Melbourne, VIC. 4 Department of Medicine, Austin Health and Northern Health, University of Melbourne, Melbourne, VIC

5 Royal Darwin Hospital, Darwin, NT.

6 Menzies School of Health Research, Darwin, NT.

7 Faculty of Health Sciences, Curtin University, Melbourne, VIC

8 Burnet Institute Melbourne, VIC.

9 School of Pathology and Laboratory Medicine, University of Western Australia, Perth, WA

10 Investigation, Response and Policy Planning and Information Sections. Communicable Disease Prevention and Control Department of Health, Victoria, Melbourne, VIC

richards@burnet.edu.au

MJA 2012; 196: 1-5 doi: 10.5694/mjall.11026 


\begin{tabular}{|c|c|}
\hline Sample & Test \\
\hline \multicolumn{2}{|l|}{ Early in the clinical course } \\
\hline \multicolumn{2}{|l|}{ Blood } \\
\hline Plasma (EDTA tube) & Flavivirus/MVEV PCR \\
\hline Serum & Serological tests \\
\hline CSF (at least $0.5 \mathrm{~mL}$ ) & $\begin{array}{l}\text { Standard microscopy and culture, Flavivirus/MVEV/ } \\
\text { HSV/enterovirus PCR, Flavivirus/MVEV serological } \\
\text { tests }\end{array}$ \\
\hline $\begin{array}{l}\text { Throat swab or nasopharyngeal aspirate } \\
\text { and faecal swabs }\end{array}$ & $\begin{array}{l}\text { Enterovirus PCR (differential diagnosis for } \\
\text { meningoencephalitis) }\end{array}$ \\
\hline \multicolumn{2}{|c|}{$\begin{array}{l}\text { During convalescence } \\
\text { ( } 7-10 \text { days later, and consider repeating } 4 \text { weeks later) }\end{array}$} \\
\hline \multicolumn{2}{|l|}{ Blood } \\
\hline Serum & Flavivirus/MVEV serological tests \\
\hline
\end{tabular}

and thus MRI does not allow discrimination between these infections. ${ }^{20-24}$ Future studies are planned to determine if MRI can be used to predict patient outcomes. Unfortunately, MRI is not available in many rural areas and computed tomography $(\mathrm{CT})$, which is more widely available, lacks the sensitivity to detect mild changes. In severe disease, CT may detect hydrocephalus, reduced attenuation from the thalami to the brainstem, cerebral atrophy or cerebral infarcts. ${ }^{16,25}$

\section{Laboratory tests}

CSF findings in people with MVEV infection will usually be consistent with a viral meningoencephalitis, comprising a raised protein concentration, normal glucose concentration and a pleomorphic leukocytosis with a predominance of mononuclear cells. ${ }^{14,16,17}$ However, data from other flavivirus encephalitides such as WNV suggest that a predominance of neutrophils may also occur. ${ }^{26}$ Laboratory confirmation of a suspected MVEV infection requires either the isolation of the virus itself, detection of MVEV RNA, a fourfold rise in the IgG titre between acute and convalescent serum samples, or detection of IgM in serum or CSF (in the absence of cross-reactive antibodies). ${ }^{16,18,27}$

The precise duration of MVEV viraemia is uncertain, but, for WNV viraemia, is less than 14 days from the time of inoculation. ${ }^{28}$ Indeed, by the time neurological symptoms develop, PCR testing of serum or CSF often returns a negative result. ${ }^{20,29-32}$ The use of PCR in conjunction with serological tests can increase the sensitivity of WNV diagnostic tests to $94 \%$ compared with $45 \%$ for serological tests alone. ${ }^{33}$ Antigen detection assays are not available for MVEV, but have been used for JEV and Dengue fever virus. $^{34,35}$

Serological tests remain the mainstay of confirmatory diagnostic testing. Overseas experience with WNV and JEV encephalitis suggests that IgM in serum usually appears 4-9 days after disease onset and can persist for months. ${ }^{16,28}$ This situation is thought to be similar for MVEV but there are limited published data. ${ }^{29,37}$ Most reference laboratories have developed in-house MVEV serological tests that can detect total antibody by haemag- glutination inhibition, IgG by immunofluorescence assays (IFA) or enzyme immunoassays (EIA), and IgM by IFA or EIA. However, cross-reactive antibodies remain problematic, and may complicate the ability to distinguish between MVEV and other flaviviruses. ${ }^{11}$ It is also possible that prior immunisation with yellow fever virus or JEV vaccine may induce cross-reactive antibodies. ${ }^{38}$ Neutralisation tests or an epitope-blocking ELISA can help identify MVEV-specific antibodies. ${ }^{11,39}$ Recommended tests for suspected MVEV are outlined in Box 2.

\section{Treatment}

Treatment of MVEV remains supportive, as there are no therapeutic options of proven clinical value. Thus, early transfer to a centre with intensive care facilities is essential. Opportunities to assess potential treatments have been limited because of low numbers of patients and the sporadic nature of cases distributed over a vast geographical area. Experience with related flaviviruses, especially JEV and $\mathrm{WNV}$, have provided further insights, but clear treatment options have not been identified for these infections either.

Corticosteroids are potentially beneficial in reducing intracranial pressure and modulating immune-mediated pathology. They have been administered to patients with MVEV, but have not been assessed in controlled studies. Although glucocorticoids have been shown to increase WNV viraemia in dogs, ${ }^{40}$ there is no evidence that they worsen outcomes in humans. Dexamethasone was assessed in a randomised control trial of 65 patients with JEV and no benefit or detriment was observed. ${ }^{41}$ Therefore, corticosteroids cannot be recommended for the definitive treatment of flavivirus encephalitis, but may still be appropriate for the reduction of intracranial pressure.

No antiviral drugs have been trialled in the treatment of humans with MVEV and there are few in-vitro or animalmodel data. There have also been few studies of antiviral drugs for other flaviviruses. Ribavirin (already established as therapy for hepatitis C virus, which is also in the Flaviviridae family) was used in a study of 153 Indian children with JEV encephalitis, but did not indicate any benefit compared with placebo. ${ }^{42}$ Interferon alfa-2a has also been assessed with a randomised, placebo-controlled study in JEV encephalitis, and failed to show any benefit compared with placebo in 112 Vietnamese children. ${ }^{43}$ The role of antiviral drugs for JEV has recently been reviewed. ${ }^{44}$

The role of immunoglobulin therapy is also unclear. In 1917, a 12-year-old with features suggestive of MVEV infection recovered after receiving immune serum. ${ }^{45}$ There is also a report of a man with JEV recovering after being administered non-immune intravenous immunoglobulin. ${ }^{46}$ There are numerous reports of immunotherapy for WNV encephalitis in Israel and the United States with WNV-specific intravenous immunoglobulin (Omr-IgGam, Omrix Biopharmaceuticals, Tel Aviv, Israel) obtained from donors with high titres of WNV antibodies. Results have been variable, but a number of favourable outcomes have been described. ${ }^{47,48}$ There is further evidence for the benefit of intravenous immunoglobulin therapy in WNV animal models, but only if administered early in the course 
of the disease. ${ }^{49}$ Phase I/II clinical trials of WNV-specific intravenous immunoglobulin therapy have recently been completed in the US (NCT00069316), but results are yet to be reported. Animal models of MVEV have shown that IgG from humans and mice exposed to MVEV can protect mice from symptomatic disease if they are transfused before virus inoculation. ${ }^{50}$ The efficacy of intravenous immunoglobulin therapy may not only be limited by timing, but by penetration into the central nervous system and its effect on intraneuronal virus. ${ }^{47}$

In light of these data, we cannot currently recommend any specific antiviral therapy for infection with flavivirus encephalitides, including MVEV, and the complex pathogenesis of this disease means that there is a potential for harm with some interventions (discussed below). Therefore, we eagerly await further trial data on interventions and the future development of effective antiviral agents. Even with effective interventions, one of the challenges in evaluating and using therapeutic agents against flavivirus encephalitides is the early identification of cases and initiation of treatment before significant neurological impairment develops.

\section{Prevention}

There is currently no effective vaccination against MVEV infection, and prevention relies on the use of sentinel chicken flocks to provide a warning of virus activity, and subsequent mosquito control. Interestingly, there are animal data indicating the potential importance of crossreactive responses in protection with a preclinical live chimeric JEV vaccine (ChimeriVax-JE; Sanofi Pasteur, Lyon, France) used to induce protection against JEV, MVEV and WNV in mice. ${ }^{38}$ Such protective responses have not been observed for currently available human vaccines for JEV or yellow fever virus. ${ }^{38,50}$ The potential detriment of induced responses must also be considered, whether induced by immunisation or natural exposure. Indeed, there is evidence from mouse studies of MVEV infection being enhanced after vaccination with inactivated JEV vaccines. ${ }^{38,50}$ While an MVEV-specific vaccine is unlikely to be developed commercially, because of the low burden of disease and financial constraints, there may be a future role for the use of a chimeric JEV vaccine to provide broad protection against flavivirus infection, including MVEV. However, further studies are needed.

\section{Conclusions}

The recent activity of MVEV across Australia decreased with the arrival of winter, but the risk during this summer and the coming autumn remains uncertain, especially in areas that remain flooded. Recent circumstances remind us of the limited information we have about this disease, the challenges of clinical management, and the need to prepare for future outbreaks. In addition to ongoing surveillance of mosquitoes, virus activity, and the impact of public health responses, further research is needed to improve the capacity for early diagnosis, to identify effective treatments and to improve preventive strategies.

Competing interests: No relevant disclosures.
Provenance: Not commissioned; externally peer reviewed.

1 Miles JAR, Fowler MC, Howes DW. Isolation of a virus from encephalitis in South Australia: a preliminary report. Med J Aust 1951; 1: 799-780.

2 Mackenzie JS, Broom AK. Australian X disease, Murray Valley encephalitis and the French connection. Vet Microbiol 1995; 46: 79-90.

3 Bennett N. Murray Valley encephalitis: indeed a "mysterious disease". Vic Infect Dis Bull 2008; 11: 94-107.

4 Smith DW, Speers DJ, Mackenzie JS. The viruses of Australia and the risk to tourists. Travel Med Infect Dis 2011; 9: 113-125.

5 Mackenzie JS, Lindsay MD, Coelen RJ, et al. Arboviruses causing human disease in the Australasian zoogeographic region. Arch Virol 1994; 136: 447-467.

6 Anderson SG, Donnelley M, Stevenson WJ, et al. Murray-Valley encephalitis; surveys of human and animal sera. Med J Aust 1952; 1: 110-114.

7 French EL, Anderson SG, Price AV, et al. Murray Valley encephalitis in New Guinea. I. Isolation of Murray Valley encephalitis virus from the brain of a fatal case of encephalitis occurring in a Papuan native. Am J Trop Med Hyg 1957; 6: 827-834.

8 Forbes JA. Murray Valley encephalitis 1974 - also, the epidemic variance since 1914 and predisposing rainfall patterns. Sydney: Australian Medical Publishing Company, 1978.

9 Nicholls N. A method for predicting Murray Valley encephalitis in southeast Australia using the Southern Oscillation. Aust J Exp Biol Med Sci 1986; 64: 587-594.

10 Australian Government Department of Health and Ageing. National Notifiable Diseases Surveillance System. Canberra: 2011. http://www9.health.gov.au/ cda/source/cda-index.cfm (accessed July 2011).

11 Hall RA, Broom AK, Hartnett AC, et al. Immunodominant epitopes on the NSI protein of MVE and KUN viruses serve as targets for a blocking ELISA to detect virus-specific antibodies in sentinel animal serum. J Virol Methods 1995; 51 : 201-210.

12 Broom AK, Lindsay MD, Plant AJ, et al. Epizootic activity of Murray Valley encephalitis virus in an Aboriginal community in the southeast Kimberley region of Western Australia: results of cross-sectional and longitudinal serologic studies. Am J Trop Med Hyg 2002; 67: 319-323.

13 Anderson SG. Murray Valley encephalitis; epidemiological aspects. Med J Aust 1952; 1: 97-100.

14 Bennett NM. Murray Valley encephalitis, 1974: clinical features. Med J Aust 1976; 2: 446-450.

15 Robertson EG, McLorinan H. Murray Valley encephalitis; clinical aspects. Med $\mathrm{J}$ Aust 1952; 1: 103-107.

16 Mackenzie JS, Smith DW, Broom AK, et al. Australian encephalitis in Western Australia, 1978-1991. Med J Aust 1993; 158: 591-595.

17 Burrow JN, Whelan PI, Kilburn CJ, et al. Australian encephalitis in the Northern Territory: clinical and epidemiological features, 1987-1996. Aust N Z J Med 1998; 28: 590-596.

18 Cordova SP, Smith DW, Broom AK, et al. Murray Valley encephalitis in Western Australia in 2000, with evidence of southerly spread. Commun Dis Intell 2000; 24: 368-372.

19 Solomon T, Vaughn DW. Pathogenesis and clinical features of Japanese encephalitis and West Nile virus infections. Curr Top Microbiol Immunol 2002: 267: 171-194.

20 Wong SH, Smith DW, Fallon MJ, et al. Murray valley encephalitis mimicking herpes simplex encephalitis. J Clin Neurosci 2005; 12: 822-824.

21 Einsiedel L, Kat E, Ravindran J, et al. MR findings in Murray Valley encephalitis AJNR Am J Neuroradiol 2003; 24: 1379-1382.

22 Kalita J, Misra UK. Comparison of CT scan and MRI findings in the diagnosis of Japanese encephalitis. J Neurol Sci 2000; 174: 3-8.

23 Kastrup O, Wanke I, Maschke M. Neuroimaging of infections. NeuroRx 2005; 2: 324-332.

24 Agid R, Ducreux D, Halliday WC, et al. MR diffusion-weighted imaging in a case of West Nile virus encephalitis. Neurology 2003; 61: 1821-1823.

25 Kienzle N, Boyes L. Murray Valley encephalitis: case report and review of neuroradiological features. Australas Radiol 2003; 47: 61-63.

26 Tyler KL, Pape J, Goody RJ, et al. CSF findings in 250 patients with serologically confirmed West Nile virus meningitis and encephalitis. Neurology 2006; 66: 361-365.

27 Broome A, McKinnon M, Miller M, et al. Interim national guidelines for the prevention, management and control of Murray Valley encephalitis. Communicable Diseases Network Australia. Canberra: Australian Government Department of Health and Ageing, 2005. http://www.health.gov.au/internet/ main/publishing.nsf/Content/health-arbovirus-mve-guidelines.htm (accessed Dec 2011).

28 Busch MP, Kleinman SH, Tobler LH, et al. Virus and antibody dynamics in acute west nile virus infection. J Infect Dis 2008; 198: 984-993.

29 McMinn PC, Carman PG, Smith DW. Early diagnosis of Murray Valley encephalitis by reverse transcriptase-polymerase chain reaction. Pathology 2000; 32: 49-51.

30 Stich A, Gunther S, Drosten C, et al. Clinical and laboratory findings on the first imported case of Murray Valley encephalitis in Europe. Clin Infect Dis 2003; 37: el9-e21. 
31 Hills S. Murray Valley encephalitis in Mt Isa, north Queensland. Tropical Public Health Unit Network, Queensland Health. Commun Dis Intell 2001; 25: 48.

32 Brown A, Bolisetty S, Whelan P, et al. Reappearance of human cases due to Murray Valley encephalitis virus and Kunjin virus in central Australia after an absence of 26 years. Commun Dis Intell 2002; 26: 39-44.

33 Tilley PA, Fox JD, Jayaraman GC, et al. Nucleic acid testing for West Nile virus RNA in plasma enhances rapid diagnosis of acute infection in symptomatic patients. J Infect Dis 2006; 193: 1361-1364.

34 Kumar JS, Parida M, Rao PV. Monoclonal antibody-based antigen capture immunoassay for detection of circulating non-structural protein NSI: implications for early diagnosis of Japanese encephalitis virus infection. J Med Virol 2011; 83: 1063-1070.

35 Alcon S, Talarmin A, Debruyne M, et al. Enzyme-linked immunosorbent assay specific to Dengue virus type 1 nonstructural protein NSI reveals circulation of the antigen in the blood during the acute phase of disease in patients experiencing primary or secondary infections. J Clin Microbiol 2002; 40 : 376-381.

36 Chanama S, Sukprasert W, Sa-ngasang A et al Detection of Japanese encephalitis (JE) virus-specific lgM in cerebrospinal fluid and serum samples from JE patients. Jpn J Infect Dis 2005; 58: 294-296.

37 Doherty RL, Carley JG, Filippich C, et al. Murray Valley encephalitis in Australia, 1974: antibody response in cases and community. Aust NZ J Med 1976; 6: 446-453.

38 Lobigs M, Larena M, Alsharifi M, et al. Live chimeric and inactivated Japanese encephalitis virus vaccines differ in their cross-protective values against Murray Valley encephalitis virus. J Virol 2009; 83: 2436-2445.

39 Hawkes RA, Roehrig JT, Boughton CR, et al. Defined epitope blocking with Murray Valley encephalitis virus and monoclonal antibodies: laboratory and field studies. J Med Virol 1990; 32: 31-38.
40 Bowen RA, Rouge MM, Siger L, et al. Pathogenesis of West Nile virus infection in dogs treated with glucocorticoids. Am J Trop Med Hyg 2006; 74: 670-673.

41 Hoke CH Jr, Vaughn DW, Nisalak A, et al. Effect of high-dose dexamethasone on the outcome of acute encephalitis due to Japanese encephalitis virus. J Infect Dis 1992; 165: 631-637.

42 Kumar R, Tripathi P, Baranwal M, et al. Randomized, controlled trial of oral ribavirin for Japanese encephalitis in children in Uttar Pradesh, India. Clin Infect Dis 2009; 48: 400-406.

43 Solomon T, Dung NM, Wills B, et al. Interferon alfa-2a in Japanese encephalitis: a randomised double-blind placebo-controlled trial. Lancet 2003: 361: 821826.

44 Gould EA, Solomon T, Mackenzie JS. Does antiviral therapy have a role in the control of Japanese encephalitis? Antiviral Res 2008; 78: 140-149.

45 Burnell GH. The Broken Hill epidemic. Med J Aust 1917; 2: 157-161.

46 Caramello P, Canta F, Balbiano R, et al. Role of intravenous immunoglobulin administration in Japanese encephalitis. Clin Infect Dis 2006; 43: 1620-1621.

47 Agrawal AG, Petersen LR. Human immunoglobulin as a treatment for West Nile virus infection. J Infect Dis 2003; 188: 1-4.

48 Makhoul B, Braun E, Herskovitz M, et al. Hyperimmune gammaglobulin for the treatment of West Nile virus encephalitis. Isr Med Assoc J 2009; 11: 151-153.

49 Ben-Nathan D, Lustig S, Tam G, et al. Prophylactic and therapeutic efficacy of human intravenous immunoglobulin in treating West Nile virus infection in mice. J Infect Dis 2003; 188: 5-12.

50 Broom AK, Wallace MJ, Mackenzie JS, et al. Immunisation with gamma globulin to Murray Valley encephalitis virus and with an inactivated Japanese encephalitis virus vaccine as prophylaxis against Australian encephalitis: evaluation in a mouse model.J Med Viro 2000; 61:259-265. 